\title{
A PRELIMINARY REPORT ON A MODIFIED FRIEDMAN PREGNANCY TEST
}

\author{
BY \\ A. A. KINNEAR \\ From the Union Health Department Laboratories, Capetown
}

(RECEIVED FOR PUBLICATION AUGUST 18, 1950)

The Friedman (1939) test for pregnancy has the minor disadvantages that skill is required for the intravenous injection into the ear vein of the rabbit and that some $3 \%$ (Albert, 1949; Evans and Krajian, 1944) of urine samples are toxic to the rabbit if injected intravenously. Ducey (1950) overcame some of these difficulties by injecting urine subcutaneously in doses of 20 to $30 \mathrm{ml}$. in the loose skin between the scapulae.

Using this subcutaneous technique the large, subcutaneous bulge of injected urine, and the not inconsiderable amount of squirming of the test animal while the urine was being injected, were striking disadvantages. Toxic manifestations were not unknown with the subcutaneous technique, especially if the urine was old and somewhat contaminated.

Friedman and Lapham (1931) found that by doubling the minimum effective dose they could produce strongly positive results, i.e., more than six corpora haemorrhagica in both ovaries. Ducey (1950) found, too, that the minimum effective dose by subcutaneous injection was twice that needed for positive results by intravenous injection.

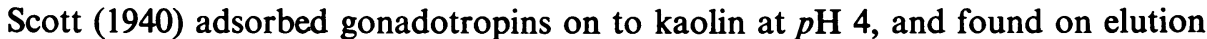
with sodium hydroxide that most if not all the toxic substances in urine remained adsorbed on the kaolin. This method, too, concentrates the gonadotropins, the concentration depending on the amount of eluent used.

To try to obviate these difficulties the following procedure was evolved.

\section{Modified Friedman Method}

The gonadotropins in $100 \mathrm{ml}$. of urine were adsorbed on kaolin at $p \mathrm{H} 4$ after the method of Scott. The gonadotropins were eluted with sodium hydroxide, and half of the neutralized extract was injected subcutaneously into each of two rabbits. With the needle still in position $1 \mathrm{mg}$. of hyaluronidase was injected from another syringe. The hyaluronidase was prepared from bull semen after the method of Gaisford and Evans (1949). After the injection of the hyaluronidase the animals soon stopped struggling and the subcutaneous bulge rapidly disappeared. No toxic reactions were noted in more than 150 tests.

A noteworthy feature was the fact that $79 \%$ of known positive urines were unequivocally positive at 24 hours, against $68 \%$ positive by the intravenous route and $59 \%$ positive by the subcutaneous route. This is presumably due to the higher 
concentration of gonadotropins and the more rapid absorption due to the hyaluronidase. The Friedman test should still be read as a routine at 48 hours before a negative report is issued.

\section{REFERENCES}

Albert, A. (1949). Proc. Mayo Clin., 24, 259.

Ducey, E. F. (1950). Amer. J. clin. Path., 20, 289.

Evans, N., and Krajian, A. A. (1944). Ibid., 14, Tech. Supp., 8, 97.

Friedman, M. H. (1939). Endocrinology, 24, 617. and Lapham, M. E. (1931). Amer. J. Obstet. Gynec., 21, 405.

Gaisford, W., and Evans, D. G. (1949). Lancet, 2, 505.

Scott, L. D. (1940). Brit. J. exp. Path., 21, 320. 